Article

\title{
Rapid Profiling of Soybean Aromatic Compounds Using Electronic Nose
}

\author{
Ramasamy Ravi ${ }^{\circledR}$, Ali Taheri * ${ }^{-}$, Durga Khandekar and Reneth Millas \\ Department of Agricultural and Environmental Sciences, Tennessee State University, 3500 John A, Merritt Blvd, \\ Nashville, TN 37209-1561, USA; rravi@tnstate.edu (R.R.); shama.khandekar@gmail.com (D.K.); \\ renz_millas@yahoo.com (R.M.) \\ * Correspondence: ataheri1@TNstate.edu
}

Received: 29 April 2019; Accepted: 20 May 2019; Published: 24 May 2019

check for updates

\begin{abstract}
Soybean (Glycine max (L.)) is the world's most important seed legume, which contributes to $25 \%$ of global edible oil, and about two-thirds of the world's protein concentrate for livestock feeding. One of the factors that limit soybean's utilization as a major source of protein for humans is its characteristic soy flavor. This off-flavor can be attributed to the presence of various chemicals such as phenols, aldehydes, ketones, furans, alcohols, and amines. In addition, these flavor compounds interact with protein and cause the formation of new off-flavors. Hence, studying the chemical profile of soybean seeds is an important step in understanding how different chemical classes interact and contribute to the overall flavor profile of the crop. In our study, we utilized the HERCALES Fast Gas Chromatography (GC) electronic nose for identification and characterization of different volatile compounds in five high-yielding soybean varieties, and studied their association with off-flavors. With aroma profiling and chemical characterization, we aim to determine the quantity and quality of volatile compounds in these soybean varieties and understand their effect on the flavor profiles. The study could help to understand soybean flavor characteristics, which in turn could increase soybean use and enhance profitability.
\end{abstract}

Keywords: e-nose; soybean; volatile; beany; electronic nose

\section{Introduction}

Soybean (Glycine max (L.)) seed protein content is $35-50 \%$ of its total dry weight and is a major source of protein in the human diet and for animal nutrition. Soybean protein also has a well-balanced amino acid profile and is rich in many essential amino acids. Soybean meal has been used extensively to make popular food products such as tofu, soy milk, soybean paste (miso), green soybeans (edamame), boiled beans (nimame), fermented soybeans (natto), soy sauce (shoyu), soybean sprouts (moyashi), and roasted soybean flour (kinako). Soybean consumption has been limited in the western world due to the beany flavor present in soy meal products. Enzymatic oxidation of linoleic acid and linolenic acid by lipoxygenase genes (Lox) is reported as a major cause of the beany flavor [1,2], and in soybeans there are three separate genes, Lox1, Lox2 and Lox3 controlling this trait [2]. Hexanal is commonly associated with the grassy flavor; hexanol; 1-octen-3-ol; 1-octen-3-one; trans,trans-2,4-decadienal; and trans,trans-2,4-nonadienal are other aromatic compounds linked with the beany taste in soy meal products [3]. Odor compounds of soybean products depend on the soybean cultivars and can change in each variety depending on growing season, storage conditions and processing technologies. Boiling the seeds at $100{ }^{\circ} \mathrm{C}$ deactivates the lipoxygenase enzymes and is the common method used for reducing the beany flavor. Breeding soybean lines with reduced beany flavor is another approach that can be used for minimizing the off-flavors in soybeans. In order to establish such breeding programs, establishing a reliable and fast screening method for testing beany flavor is necessary. Plant volatile 
compounds such as beany flavor are overlooked in plant phenotyping. Novel molecular techniques and marker assisted breeding can be used to map the QTLs controlling these traits and find the loci/genetic mechanisms regulating these compounds which can then be exploited for developing soybean lines with reduced off-flavor traits.

Various volatile compounds may serve as indicators of developmental maturity and as biochemical markers to evaluate seed quality. Several compound classes identified were alcohols, aldehydes, esters and lactones, ketones, and terpenoids. Many reports are available on the key volatiles of soybean [4-8]. The development of objectionable off-odor detection and classification methodology for use in grain grading has stimulated research on volatile components of soybeans and grains [9].

\section{Current Analytical Approaches in Volatile Compound Measurements, Especially in Seeds}

Numerous analytical approaches have been developed for measuring volatile compounds and gas exchange measurements in seed samples. Stephen et al. [10] analyzed the soybean seed volatiles using a solid phase microextraction (SPME) method combined with gas chromatography-mass spectrometry (GC-MS) and reported that 30 known volatile compounds were recovered, and that an additional 19 new compounds were identified, or tentatively identified. During early periods of development at maturity stage R6, several volatiles were present at relatively high concentrations, including 3-hexanone, (E)-2-hexenal, 1-hexanol, and 3-octanone. At maturity stage R7 and R8, decreased amounts of 3-hexanone, (E)-2-hexenal, 1-hexanol, and 3-octanone were observed. At maturity stage R8, hexanal, (E)-2-heptenal, (E)-2-octenal, ethanol, 1-hexanol, and 1-octen-3-ol were detected at relatively high concentrations.

An investigation by Shu et al. [11] using an aroma extract dilution analysis (AEDA) of the aroma concentrate of soy milk made from a major Japanese soybean cultivar, Fukuyutaka (FK), revealed 20 key aroma compounds having flavor dilution (FD) factors of not less than 64 . Among them; 2-isopropyl-3-methoxypyrazine; cis-4,5-epoxy-(E)-2-decenal; trans-4,5-epoxy-(E)-2-decenal; 3-hydroxy-4,5-dimethyl-2(5H)-furanone; and 2'-aminoacetophenone were identified as the key aroma compounds in soy milk for the first time. Generally, it is believed that aroma compounds might be generated from lipids, amino acids, sugars, and ferulic acid present in food.

Sample preparation and scalability of GC-MS and similar instruments resulted in the development of cheaper, faster, and more user-friendly measurement instruments for routine use in analytical applications. Electronic nose (e-nose) devices are developed as versatile and low-cost alternatives to GC-MS instruments that minimize sample preparation and extraction, and offer many potential uses in biomedical and agriculture applications [12]. Volatile compounds can be measured from the sample headspace with minimal sample preparation time. The objective of this study was to use an e-nose instrument in measuring the volatile compounds among five different soybean cultivars and evaluate its potential as an alternative to GC-MS approach, and as a rapid screening tool for aromatic variations in soybean seeds.

\section{Materials and Methods}

\subsection{Plant Materials}

Five recent soybean releases were selected for these experiments including, UA5014C, UA5414RR, JTN-5503, JTN-5110, and JTN-5203. These lines were reported to have a higher yielding potential in a statewide comparison and resistance to common diseases in southern states of US (Table 1). The UA5014C and UA5414RR lines were developed by the Arkansas Agricultural Experiment Station, while JTN-5503, JTN-5110, and JTN-5203 were developed at USDA-ARS Jackson Research Station. Parental information for these lines is provided in Table 1. These lines were grown at the Tennessee State University research farm in 2017. The experimental unit consisted of three replicates with two rows ( 20 feet deep) and a planting density of 5 seeds/ft. 
Table 1. List of soybean lines, plant introduction (PI) number, pedigree, maturity group (MG) and year of release.

\begin{tabular}{ccccc}
\hline Breeding Material & Plant Introduction \# & Parental Lines & MG & Reference \\
\hline JTN5503 & PI 641938 & Fowler $\times$ Manokin & V (5.5) & Arelli et al. [13] \\
JTN5110 & PI 678369 & J98-32 (Manokin $\times$ Fowler.) $\times$ Anand & V (5) & Arelli et al. [14] \\
JTN5203 & PI 664903 & Caviness $\times$ Anand & V (5) & Arelli et al. [15] \\
UA 5014C & PI 675648 & Ozark $\times$ Anand & V (5) & Chen et al. [16] \\
UA 5414RR & NA & R96-3427 $\times$ 98,601 & V (5.4) & Pengyin et al. [17] \\
\hline
\end{tabular}

\subsection{Electronic Nose}

The HERCALES GC Flash electronic nose (AlphaMos, Toulouse, France) was used to discriminate the odor patterns of different aroma models. For each variety, 20 gm of seed was weighed and grinded in a grinder (Waring WSG60 Grinder) at high speed for $2 \mathrm{~min}$. The resulting soy flour was weighed (6 gm) and placed in a $20 \mathrm{~mL}$ glass vial. Following this, $7 \mathrm{~mL}$ of sterile distilled water was added to each tube. The sample was prepared in a septa-sealed screw cap vial and equilibrated for $200 \mathrm{~s}$ at $50{ }^{\circ} \mathrm{C}$, separately. Subsequently, the aroma headspace above the sample was introduced into the electronic nose at the speed of $270 \mu \mathrm{L} / \mathrm{s}$ using automatic headspace sampler (PerkinElmer, MA, USA). The column temperature program used for the experiment was $40{ }^{\circ} \mathrm{C}(1 \mathrm{~min})-2{ }^{\circ} \mathrm{C} / \mathrm{min}-200{ }^{\circ} \mathrm{C}(3 \mathrm{~min})$, and the injection temperature of the injector and detector were set at $180^{\circ} \mathrm{C}$ and $220^{\circ} \mathrm{C}$, respectively. In addition, at the end of each column a FID detector was placed and the acquired signal was digitalized every $0.01 \mathrm{~s}$. The Heracles electronic nose is equipped with two columns working in parallel mode. A non-polar column (MXT5: 5\% diphenyl, 95\% methylpolysiloxane, $10 \mathrm{~m}$ length, and $180 \mu \mathrm{m}$ diameter), and a slightly polar column (MXT1701: 14\% cyanopropylphenyl, 86\% methylpolysiloxane, $10 \mathrm{~m}$ length, and $180 \mu \mathrm{m}$ diameter). A single comprehensive chromatogram was generated by joining the chromatograms obtained with the two columns. This approach helps reduce incorrect identifications due to overlapping of chromatograms obtained with two different columns, and represents a useful tool for improved identification. For calibration of the instrument, an alkane solution (from n-hexane to n-hexadecane) was used to convert retention time in Kovats indices and to identify the volatile compounds using specific software (AromaChemBase). Each analysis was repeated a total of three times, and all of the response data was analyzed using Alpha Soft software (Version 3.0.0, Toulouse, France).

\subsection{Results and Discussion}

The volatile profiles were generated using the e-nose and were subjected to PCA analysis. The PCA plot (Figure 1) shows the distinct clusters formed for different soy varieties indicating that the volatile profiles of soy varieties are distinctly different from each other. It also demonstrates the potential use of this system in rapid profiling of volatile compounds in different soybean cultivars. UA5414RR and UA5014C were comparable in their volatile profiles while other samples namely JTN5203, JTN5503, and JTN5110 were distantly diverse different from one another. The different clusters formed for different samples are due to their differential volatile compounds and their composition.

More than $90 \%$ of the volatile compounds were identified with Kovats index and Arochembase software in UA5014C (Figure 2). The total volatile composition is distributed between acids, aldehydes, alcohols, esters, pyrazines (Table 2 and Figure 3). However, the major volatile composition was contributed by Ethyl-2-Methyl Butyrate (22.72\%), 2-Methyl Propanal (18.21\%) and 2-Propanol (16.45\%). These three volatile components nearly contribute $50 \%$ of the total volatile composition in this cultivar. In UA5414RR (Table 3, Figures 2 and 3), the contribution of Ethyl-2-Methyl Butyrate (24.07\%) and 2-Methyl Propanal (19.42\%) is still high but instead of 2-Propanol, contribution of Ethyl 2-Methylbutanoate (16.01\%) was higher in the total volatile composition. From Figure 3, it is clear that esters were the major contributor of the volatiles followed by aldehydes and alcohols in both UA5414RR and UA5014C. Acids and monoterpenes were not detected in UA5414RR. Alcohol was significantly higher in UA5014C compared to UA5414RR. 


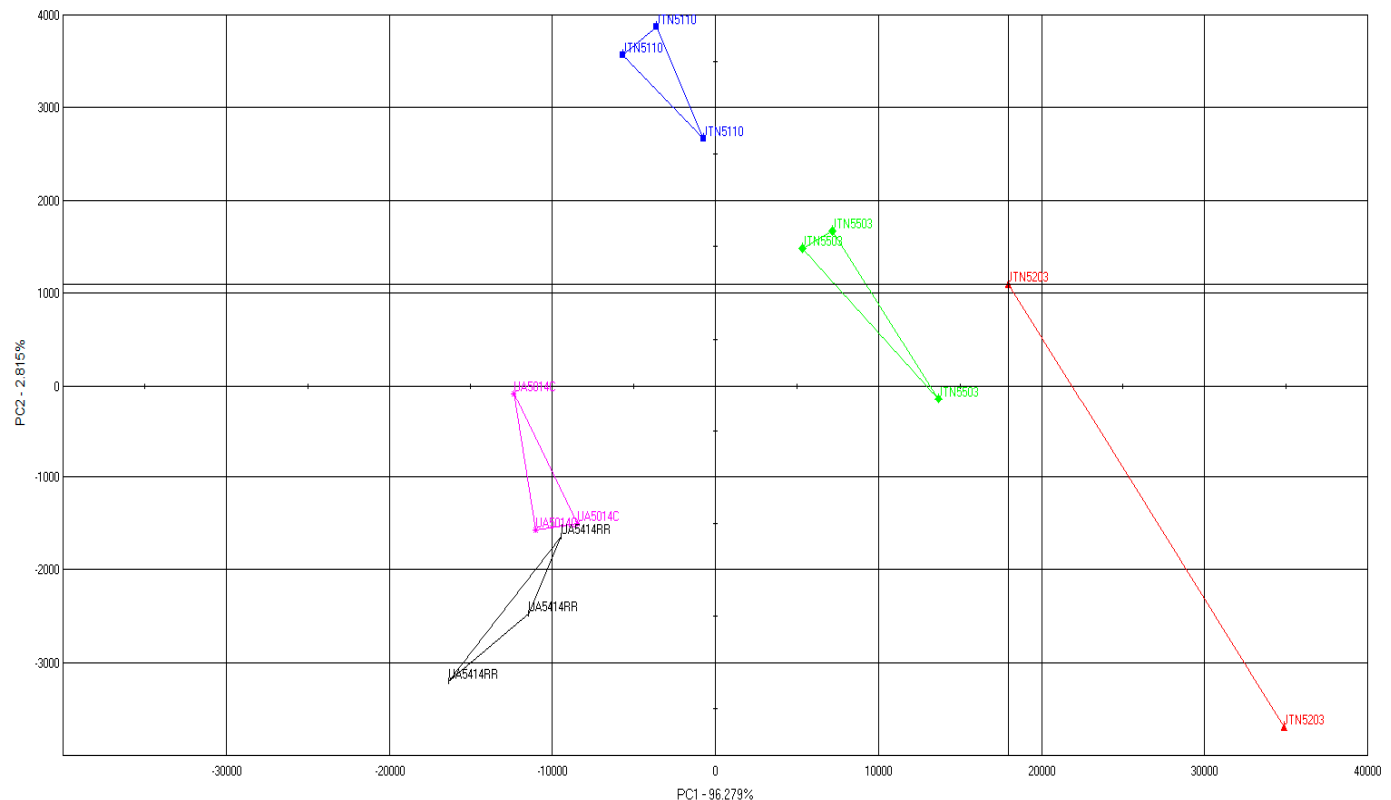

Figure 1. PCA plot of volatile profiles from soybean cultivars.

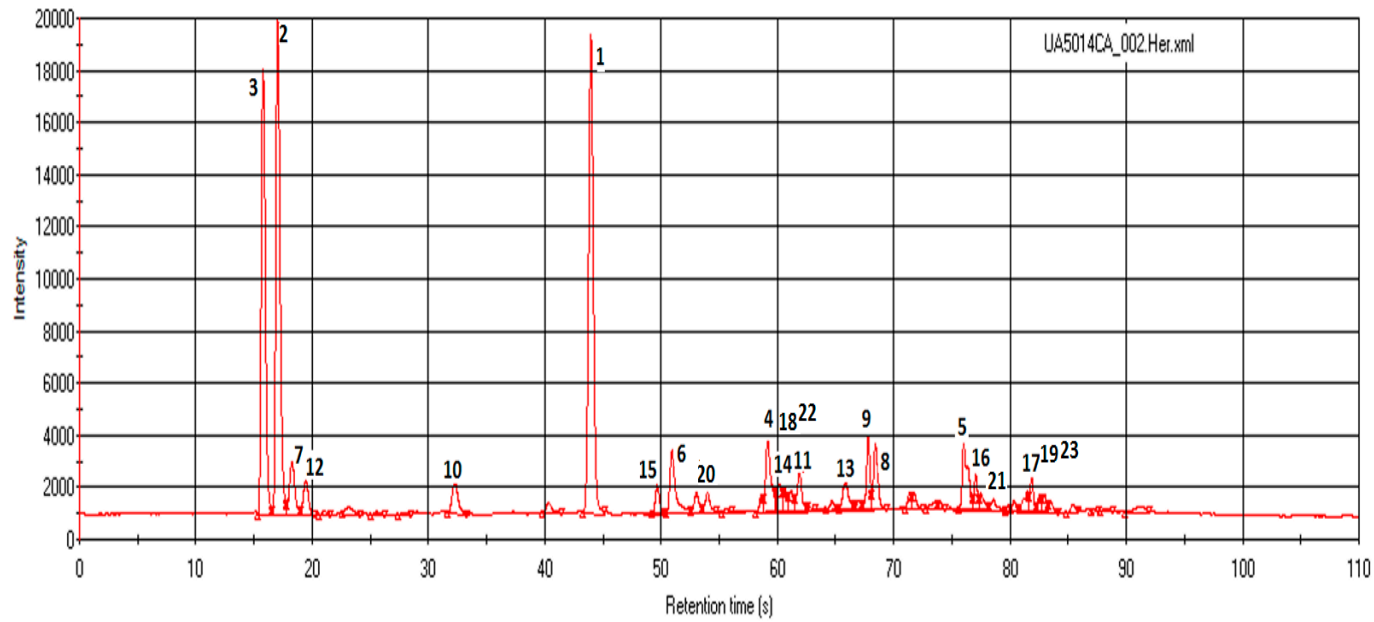

(A) UA5014C

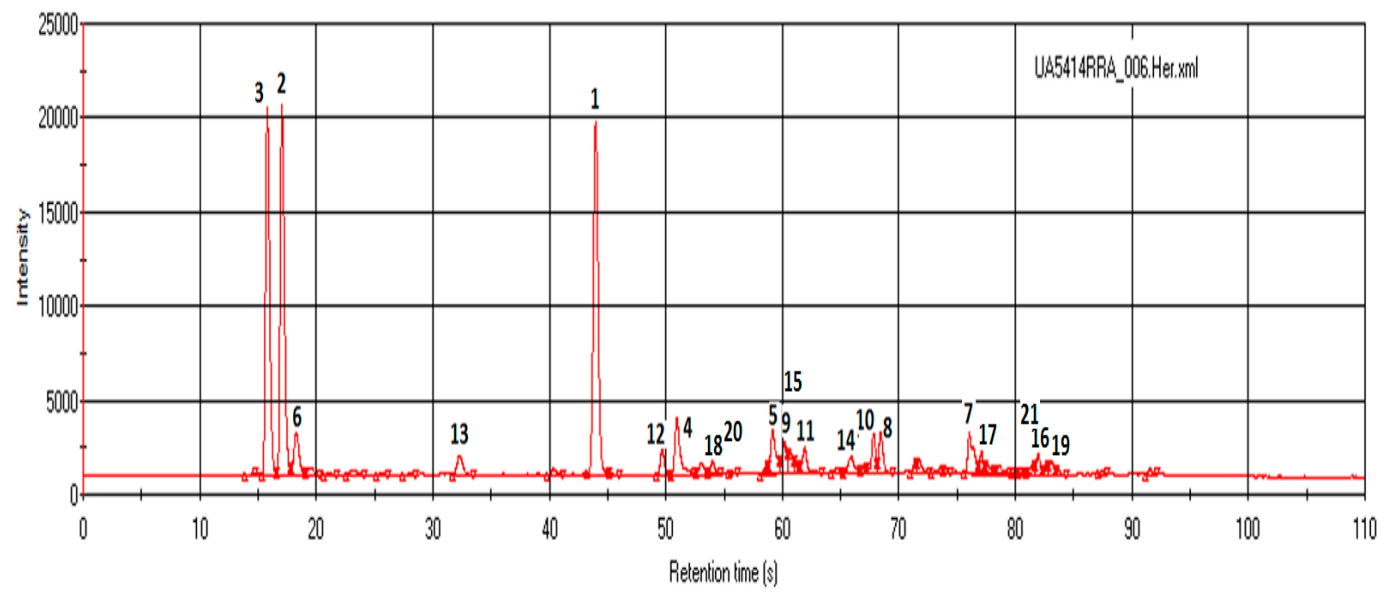

(B) UA5414RR

Figure 2. Ultra-fast GC Chromatogram of soybean varieties: UA5014C and UA5414RR. 
Table 2. Headspace volatile compounds of soybean variety: UA5014C

\begin{tabular}{|c|c|c|c|c|c|c|}
\hline \multirow[b]{2}{*}{ Peak No } & \multicolumn{4}{|c|}{ Volatile Compounds } & \multirow{2}{*}{$\begin{array}{l}\text { Retention } \\
\text { Time (s) }\end{array}$} & \multirow{2}{*}{$\begin{array}{l}\text { Kovat's } \\
\text { Index }\end{array}$} \\
\hline & Name & Surface Percent & $\begin{array}{c}\text { Category/Total } \\
\text { Percent }\end{array}$ & Sensory Descriptors & & \\
\hline 19 & Pentanoic Acid & $0.91 \pm 0.07$ & \multirow{2}{*}{ Acids 1.73} & \multirow{2}{*}{ Beefy, cheese, pungent, sour, sweaty } & 81.47 & 1366 \\
\hline 21 & Butanoic Acid & $0.82 \pm 0.06$ & & & 77.49 & 1281 \\
\hline 3 & 2-Propanol & $16.45 \pm 1.26$ & \multirow{3}{*}{ Alcohols 19.15} & Alcoholic, ethereal & 15.78 & 505 \\
\hline 12 & 2-Methyl-1-Propanol & $1.54 \pm 0.08$ & & Alcoholic, bitter, chemical, glue & 19.43 & 599 \\
\hline 15 & 3-Heptanol & $1.16 \pm 0.13$ & & Green, herbaceous & 49.68 & 881 \\
\hline 2 & 2-Methyl Propanal & $18.21 \pm 0.71$ & \multirow{7}{*}{ Aldehydes 32.35} & Burnt, fruity, green malty, pungent, spicy, toasted & 17.03 & 538 \\
\hline 4 & Benzaldehyde & $3.67 \pm 0.33$ & & Almond, burnt sugar, fruity, woody & 59.17 & 971 \\
\hline 5 & P-Anisaldehyde & $3.39 \pm 0.13$ & & Anise, minty, sweet & 76.02 & 1252 \\
\hline 7 & Butanal & $2.61 \pm 0.08$ & & Chocolate, green, malty, pungent & 18.26 & 569 \\
\hline 8 & N-Nonanal & $2.44 \pm 0.11$ & & Chlorine, citrus, fatty, floral, fruity, gaseous, gravy, green, lavender & 68.42 & 1110 \\
\hline 14 & Benzaldehyde & $1.28 \pm 0.06$ & & Almond, burnt sugar, fruity, woody & 60.19 & 984 \\
\hline 22 & 2-Decenal & $0.75 \pm 0.01$ & & Fatty, orange & 60.67 & 990 \\
\hline 1 & Ethyl-2-methyl Butyrate & $22.72 \pm 1.92$ & \multirow{5}{*}{ Esters 28.42} & Apple, blackberry, fruity, green, strawberry, sweet & 43.96 & 854 \\
\hline 9 & Ethyl Heptanoate & $2.29 \pm 0.08$ & & Grape like & 67.81 & 1099 \\
\hline 10 & Ethyl Butyrate & $1.71 \pm 0.04$ & & Acetone, banana, bubblegum, caramelized, fruity & 32.26 & 799 \\
\hline 11 & Hexyl Acetate & $1.66 \pm 0.04$ & & Acidulous, citrus, fruity, green, herbaceous, sweet wine, tobacco, rubber, spicy & 61.90 & 1007 \\
\hline 23 & Ethyl Hexanoate & $0.04 \pm 0.01$ & & Anise, apple, fruity, strawberry, sweet, winegum & 82.96 & 1399 \\
\hline 6 & 2-Heptanone & $3.22 \pm 0.10$ & \multirow{5}{*}{ Ketones 7.72} & Cheese, cured ham, fruity, gaseous, gravy, nutty, soapy & 50.93 & 887 \\
\hline 13 & Acetophenone & $1.51 \pm 0.11$ & & Almond, cheese, floral, musty, sweet & 65.87 & 1069 \\
\hline 16 & Carvone & $1.09 \pm 0.02$ & & Minty, warm, herbaceous & 77.04 & 1272 \\
\hline 17 & Delta Nonalactone & $1.05 \pm 0.05$ & & Coconut & 81.86 & 1375 \\
\hline 20 & Gamma Nonalactone & $0.85 \pm 0.09$ & & Coconut, fruity, peach, woody & 53.03 & 897 \\
\hline \multirow[t]{2}{*}{18} & Trimethyl Pyrazine & $0.95 \pm 0.08$ & Pyrazines 0.95 & Cocoa, earthy, musty, nutty, peanut, potato, roasted nut & 61.20 & 997 \\
\hline & SUM & $90.26 \pm 0.08$ & & & & \\
\hline
\end{tabular}


Table 3. Headspace volatile compounds of soybean variety: UA5414RR.

\begin{tabular}{|c|c|c|c|c|c|c|}
\hline \multirow[b]{2}{*}{ Peak No. } & \multicolumn{4}{|c|}{ Volatile Compounds } & \multirow{2}{*}{$\begin{array}{l}\text { Retention } \\
\text { Time (s) }\end{array}$} & \multirow{2}{*}{$\begin{array}{l}\text { Kovat's } \\
\text { Index }\end{array}$} \\
\hline & Name & Surface Percent & $\begin{array}{c}\text { Category/Total } \\
\text { Percent }\end{array}$ & Sensory Descriptors & & \\
\hline 12 & 3-Heptanol & $1.49 \pm 0.05$ & \multirow{2}{*}{ Alcohol 2.29} & Green, herbaceous & 49.68 & 881 \\
\hline 20 & 1-Heptanol & $0.80 \pm 0.04$ & & Green, herbaceous & 53.05 & 897 \\
\hline 2 & 2-Methyl Propanal & $19.42 \pm 1.59$ & \multirow{4}{*}{ Aldehydes 27.31} & Burnt, fruity, green malty, pungent, spicy, toasted & 17.05 & 538 \\
\hline 5 & Benzaldehyde & $3.15 \pm 0.23$ & & Almond, burnt sugar, fruity, woody & 59.16 & 970 \\
\hline 7 & p-Anisaldehyde & $2.78 \pm 0.10$ & & Anise, minty, sweet & 76.05 & 1252 \\
\hline 9 & Phenylmethanal & $1.96 \pm 0.12$ & & Almond, burnt sugar, fruity, woody & 60.20 & 984 \\
\hline 1 & Ethyl-2-Methyl Butyrate & $24.07 \pm 4.36$ & \multirow{6}{*}{ Esters 47.19} & Apple, blackberry, fruity, green, strawberry, sweet & 43.98 & 855 \\
\hline 3 & Ethyl 2-Methylbutanoate & $16.01 \pm 3.44$ & & Apple, blackberry, fruity, green, strawberry, sweet & 15.80 & 506 \\
\hline 8 & Ethyl Heptanoate & $2.11 \pm 0.10$ & & Grape like & 68.43 & 1110 \\
\hline 10 & Ethyl Enanthate & $1.94 \pm 0.10$ & & Acidic, fruity & 67.82 & 1099 \\
\hline 11 & Hexyl Acetate & $1.61 \pm 0.12$ & & Acidulous, citrus, fruity, green, herbaceous, sweet wine, tobacco, rubber, spicy & 61.92 & 1007 \\
\hline 13 & Ethyl Butyrate & $1.45 \pm 0.08$ & & Acetone, banana, bubblegum, caramelized, fruity & 32.30 & 799 \\
\hline 4 & 2-Heptanone & $4.30 \pm 0.30$ & \multirow{7}{*}{ Ketones 12.07} & Cheese, cured ham, fruity, gaseous, gravy, nutty, soapy & 50.95 & 887 \\
\hline 6 & Butane-2,3-Dione & $2.83 \pm 0.12$ & & Butter, caramelized, creamy, fruity, pineapple, spirit & 18.28 & 570 \\
\hline 14 & Acetophenone & $1.35 \pm 0.03$ & & Almond, cheese, floral, musty, sweet & 65.92 & 1069 \\
\hline 16 & Delta Nonalactone & $1.04 \pm 0.09$ & & Coconut & 81.96 & 1377 \\
\hline 17 & Carvone & $0.96 \pm 0.01$ & & Minty, warm, herbaceous & 77.08 & 1273 \\
\hline 19 & Delta Nonalactone & $0.86 \pm 0.03$ & & Coconut & 83.08 & 1401 \\
\hline 21 & $\gamma$-Nonalactone & $0.73 \pm 0.01$ & & Coconut, fruity, peach, woody & 81.57 & 1368 \\
\hline 15 & ß-Pinene & $1.21 \pm 0.06$ & Monoterpens 1.21 & Terpenic & 60.68 & 990 \\
\hline \multirow[t]{2}{*}{18} & 2,5-Dimethyl Pyrazine & $0.89 \pm 0.02$ & Pyrazines 0.89 & Chocolate, cocoa, medicinal, roast beef, roasted nut, woody & 54.03 & 905 \\
\hline & SUM & \multicolumn{2}{|c|}{90.94} & & & \\
\hline
\end{tabular}




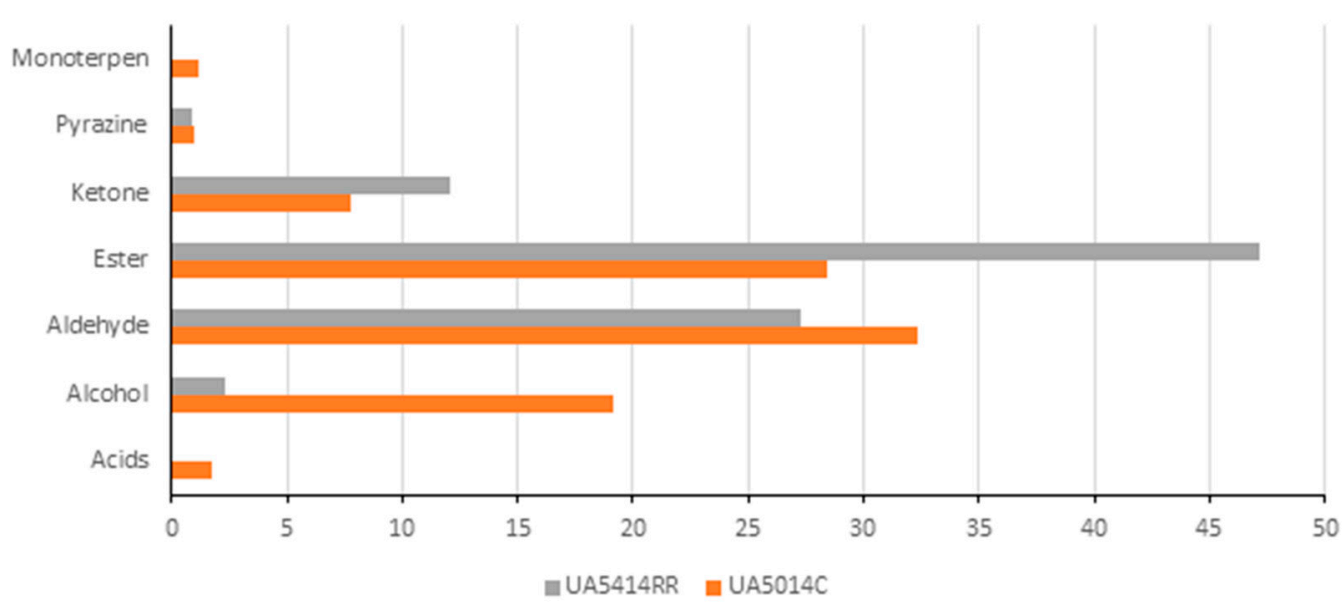

Figure 3. Comparison of volatile compounds in soybean varieties: UA5014C and UA5414RR.

In JTN5503 (Table 4, Figures 4 and 5), Ethyl formate (48.29) and Ethyl-2-Methyl Butyrate (10.12) presence was higher than other compounds whereas in JTN5110 (Table 5, Figures 4 and 5), Dimethyl sulphide (34.2) and Ethyl-2-Methyl Butyrate (14.39) were higher. In JTN5203 (Table 6, Figure 4), Dimethyl sulphide alone contributed to over $64 \%$ of the total volatile composition. A visual comparison of the peaks in Figure 4 clearly indicates the differences between JTN cultivars in peaks 2, 5, and 17. From Figure 5, it is clear that esters were the major contributor of the volatiles followed by aldehydes and ketones in JTN5203, JTN5503 and JTN5110. The sulfur containing compounds were a major volatile contributor in JTN5110, and JTN5203 but not in JTN5503. Furans were detected only in JTN5110 and were absent in the other two varieties. In general, acids, furans and pyrazines were low in all the samples.

PCA analysis indicated that UA5414RR and UA5014C were comparable in their volatile profiles while other samples namely JTN5203, JTN5503, and JTN5110 were distantly different from each other (Figure 1). Different clusters formed in different samples according to their differential volatile compounds and their compositions (Tables 2-6). It should be noted that beany flavor is caused by a combination of different compounds and assigning specific flavor to a cultivar should be carried out using sensory analysis with a panel of trained evaluators. 
Table 4. Headspace volatile compounds of soybean variety: JTN5503.

\begin{tabular}{|c|c|c|c|c|c|c|}
\hline \multirow[b]{2}{*}{ Peak No. } & \multicolumn{4}{|c|}{ Volatile Compounds } & \multirow{2}{*}{$\begin{array}{l}\text { Retention } \\
\text { Time (s) }\end{array}$} & \multirow{2}{*}{$\begin{array}{l}\text { Kovat's } \\
\text { Index }\end{array}$} \\
\hline & Name & Surface Percent & $\begin{array}{c}\text { Category/Total } \\
\text { Percent }\end{array}$ & Sensory Descriptors & & \\
\hline 13 & Methyl Eugenol & $1.15 \pm 0.07$ & \multirow{2}{*}{ Alcohol 1.9} & Clove, spicy. & 83.00 & 1400 \\
\hline 19 & 3-Heptanol & $0.75 \pm 0.04$ & & Green, herbaceous & 53.04 & 897 \\
\hline 5 & Benzaldehyde & $2.51 \pm 0.11$ & \multirow{5}{*}{ Aldehyde 9.3} & Almond, burnt sugar, fruity, woody & 59.15 & 970 \\
\hline 6 & Butanal & $2.43 \pm 0.14$ & & Chocolate, green, malty, pungent & 18.21 & 568 \\
\hline 7 & N-Nonanal & $1.86 \pm 0.16$ & & Chlorine, citrus, fatty, floral, fruity, gaseous, gravy, green, lavender & 68.44 & 1110 \\
\hline 10 & P-Anisaldehyde & $1.28 \pm 0.08$ & & Anise, minty, sweet & 76.06 & 1253 \\
\hline 11 & Benzaldehyde & $1.22 \pm 0.07$ & & Almond, burnt sugar, fruity, woody & 60.19 & 984 \\
\hline 1 & Ethyl Formate & $48.29 \pm 3.85$ & \multirow{6}{*}{ Ester 70.21} & Smell of rum, Ethereal, pungent & 16.98 & 536 \\
\hline 2 & Ethyl-2-Methyl Butyrate & $10.12 \pm 1.14$ & & Apple, blackberry, fruity, green, strawberry, sweet & 43.97 & 855 \\
\hline 3 & Methyl Formate & $7.76 \pm 0.58$ & & Ethereal, pungent & 15.75 & 505 \\
\hline 8 & Ethyl Heptanoate & $1.60 \pm 0.12$ & & Grape like & 67.83 & 1099 \\
\hline 9 & Hexyl Acetate & $1.35 \pm 0.06$ & & Acidulous, citrus, fruity, green, herbaceous, sweet wine, tobacco, rubber, spicy & 61.91 & 1007 \\
\hline 15 & Ethyl Butyrate & $1.09 \pm 0.06$ & & Acetone, banana, bubblegum, caramelized, fruity & 32.26 & 799 \\
\hline 4 & 2-Heptanone & $4.38 \pm 0.63$ & \multirow{5}{*}{ Ketone 8.41} & Cheese, cured ham, fruity, gaseous, gravy, nutty, soapy & 50.93 & 887 \\
\hline 12 & Acetophenone & $1.19 \pm 0.05$ & & Almond, cheese, floral, musty, sweet & 65.91 & 1069 \\
\hline 14 & (+)-Carvone & $1.13 \pm 0.06$ & & Caraway, minty, peppermint & 76.38 & 1259 \\
\hline 16 & Gamma Nonalactone & $0.87 \pm 0.03$ & & Coconut, fruity, peach, woody & 81.88 & 1375 \\
\hline 17 & (-)-Carvone & $0.84 \pm 0.02$ & & Caraway, minty, peppermint & 77.09 & 1273 \\
\hline 18 & 2,5-Dimethyl Pyrazine & $0.74 \pm 0.09$ & Pyrazines 0.74 & Chocolate, cocoa, medicinal, roast beef, roasted nut, woody & 53.99 & 904 \\
\hline & SUM & & & & & \\
\hline
\end{tabular}


Table 5. Headspace volatile compounds of soybean variety: JTN5110.

\begin{tabular}{|c|c|c|c|c|c|c|}
\hline \multirow[b]{2}{*}{ Peak No } & \multicolumn{4}{|c|}{ Volatile Compounds } & \multirow{2}{*}{$\begin{array}{l}\text { Retention } \\
\text { Time (s) }\end{array}$} & \multirow{2}{*}{$\begin{array}{l}\text { Kovat's } \\
\text { Index }\end{array}$} \\
\hline & Name & $\begin{array}{c}\text { Surface } \\
\text { Percentage }\end{array}$ & $\begin{array}{c}\text { Category/Total } \\
\text { Percent }\end{array}$ & Sensory Descriptors & & \\
\hline 20 & Pentanoic Acid & $0.83 \pm 0.05$ & Acids 0.83 & Beefy, cheese, pungent, sour, sweaty & 53.99 & 904 \\
\hline 17 & 3-Heptanol & $0.95 \pm 0.03$ & \multirow{2}{*}{ Alcohol 2.29} & Green, herbaceous & 46.69 & 881 \\
\hline 13 & Methyl Eugenol & $1.34 \pm 0.07$ & & Clove, spicy & 82.95 & 1398 \\
\hline 18 & 2-Decenal & $0.92 \pm 0.05$ & \multirow{6}{*}{ Aldehyde 19.5} & Fatty, orange & 77.04 & 1272 \\
\hline 6 & Benzaldehyde & $2.94 \pm 0.19$ & & Almond, burnt sugar, fruity, woody & 59.17 & 971 \\
\hline 12 & Phenylmethanal & $1.39 \pm 0.08$ & & Burnt sugar, fruity, woody & 60.19 & 984 \\
\hline 7 & Butanal & $2.45 \pm 0.14$ & & Chocolate, green, malty, pungent & 18.25 & 569 \\
\hline 11 & P-Anisaldehyde & $1.45 \pm 0.09$ & & Anise, minty, sweet & 76.02 & 1252 \\
\hline 3 & Propanal & $10.35 \pm 0.33$ & & Ethereal, plastic, pungent, solvent & 15.76 & 505 \\
\hline 15 & Ethyl Butyrate & $1.28 \pm 0.06$ & \multirow{4}{*}{ Ester 18.5} & Acetone, banana, bubblegum, caramelized, fruity & 32.29 & 799 \\
\hline 16 & Mthyl Butyrate & $1.25 \pm 0.10$ & & Banana, bubblegum, caramelized, fruity & 65.92 & 1069 \\
\hline 2 & Ethyl-2-Methyl Butyrate & $14.39 \pm 1.63$ & & Apple, blackberry, fruity, green, strawberry, sweet & 43.98 & 855 \\
\hline 10 & Hexyl Acetate & $1.58 \pm 0.09$ & & Acidulous, citrus, fruity, green, herbaceous, sweet wine, tobacco, rubber, spicy & 61.92 & 1007 \\
\hline 5 & Furfural & $3.12 \pm 0.40$ & Furans 3.12 & Almond, bread, sweet & 37.26 & 823 \\
\hline 14 & (-)-Carvone & $1.30 \pm 0.06$ & \multirow{5}{*}{ Ketone 10.86} & Caraway, minty, peppermint & 76.35 & 1258 \\
\hline 4 & 2-Heptanone & $4.80 \pm 0.36$ & & Cheese, cured ham, fruity, gaseous, gravy, nutty, soapy & 50.95 & 887 \\
\hline 19 & Delta Nonalactone & $0.88 \pm 0.06$ & & Coconut & 81.84 & 1374 \\
\hline 8 & Ethyl Heptanoate & $2.09 \pm 0.12$ & & Grape like & 68.43 & 1110 \\
\hline 9 & Ethyl enanthate & $1.79 \pm 0.12$ & & Pleasant, floral & 97.84 & 1099 \\
\hline 21 & Trimethyl Pyrazine & $0.80 \pm 0.03$ & Pyrazine 0.8 & Cocoa, earthy, musty, nutty, peanut, potato, roasted nut & 60.67 & 990 \\
\hline 1 & Dimethyl Sulphide & $34.20 \pm 3.31$ & Sulfur 34.2 & Cabbage, fruity, gaseous, gasoline, moldy, vegetable soup & 17.01 & 537 \\
\hline
\end{tabular}


Table 6. Headspace volatile compounds of soybean variety: JTN 5203.

\begin{tabular}{|c|c|c|c|c|c|c|}
\hline Peak No. & Volatile Compounds & $\begin{array}{c}\text { Surface } \\
\text { Percentage }\end{array}$ & Category & Sensory Descriptors & $\begin{array}{c}\text { Retention } \\
\text { Time (s) }\end{array}$ & $\begin{array}{c}\text { Kovat's } \\
\text { Index }\end{array}$ \\
\hline 15 & Butanoic Acid & $0.82 \pm 0.02$ & Acids 0.82 & Butter, cheese, rancid, sweaty & 65.87 & 1069 \\
\hline 2 & 2-Propanol & $5.84 \pm 0.63$ & \multirow{3}{*}{ Alcohol 7.93} & Alcoholic, ethereal & 15.57 & 505 \\
\hline 10 & 3-Heptanol & $1.04 \pm 0.16$ & & 3-Heptanol & 75.96 & 1251 \\
\hline 13 & Methyl Eugenol & $1.05 \pm 0.02$ & & Clove, spicy & 76.29 & 1257 \\
\hline 5 & Benzaldehyde & $2.13 \pm 0.41$ & \multirow{5}{*}{ Aldehyde 7.28} & Almond, burnt sugar, fruity, woody & 18.23 & 568 \\
\hline 6 & Phenylmethanal & $1.46 \pm 0.15$ & & Burnt sugar, fruity, woody & 59.12 & 970 \\
\hline 7 & N-Nonanal & $1.37 \pm 0.10$ & & Chlorine, citrus, fatty, floral, fruity, gaseous, gravy, green, lavender & 60.16 & 983 \\
\hline 8 & Nonanaldehyde & $1.21 \pm 0.15$ & & Chlorine, citrus, fatty, floral, fruity, gaseous, gravy, green, lavender & 68.38 & 1109 \\
\hline 9 & p-Anisaldehyde & $1.11 \pm 0.22$ & & Anise, minty, sweet & 67.77 & 1098 \\
\hline 3 & Ethyl-2-Methyl Butyrate & $5.26 \pm 0.70$ & \multirow{3}{*}{ Ester 7.24} & Apple, blackberry, fruity, green, strawberry, sweet & 48.81 & 889 \\
\hline 11 & 2,3-Hexen-1-Ol, Acetate & $1.01 \pm 0.13$ & & Banana, fruity, green, sweet, sharp & 50.95 & 887 \\
\hline 12 & Phenyl Ethyl Acetate & $0.97 \pm 0.16$ & & Phenyl Ethyl Acetate & 61.86 & 1006 \\
\hline 4 & Butane-2,3-Dione & $3.52 \pm 0.37$ & \multirow{2}{*}{ Ketone 4.5} & Butter, caramelized, creamy, fruity, pineapple, spirit & 43.97 & 855 \\
\hline 14 & Acetophenone & $0.98 \pm 0.01$ & & Almond, cheese, floral, musty, sweet & 82.86 & 1397 \\
\hline \multirow[t]{2}{*}{1} & Dimethyl Sulphide & $64.14 \pm 3.91$ & Sulfur 64.14 & Cabbage, fruity, gaseous, gasoline, moldy, vegetable soup & 16.97 & 536 \\
\hline & Sum & \multicolumn{2}{|c|}{$91.92 \pm 0.18$} & & & \\
\hline
\end{tabular}



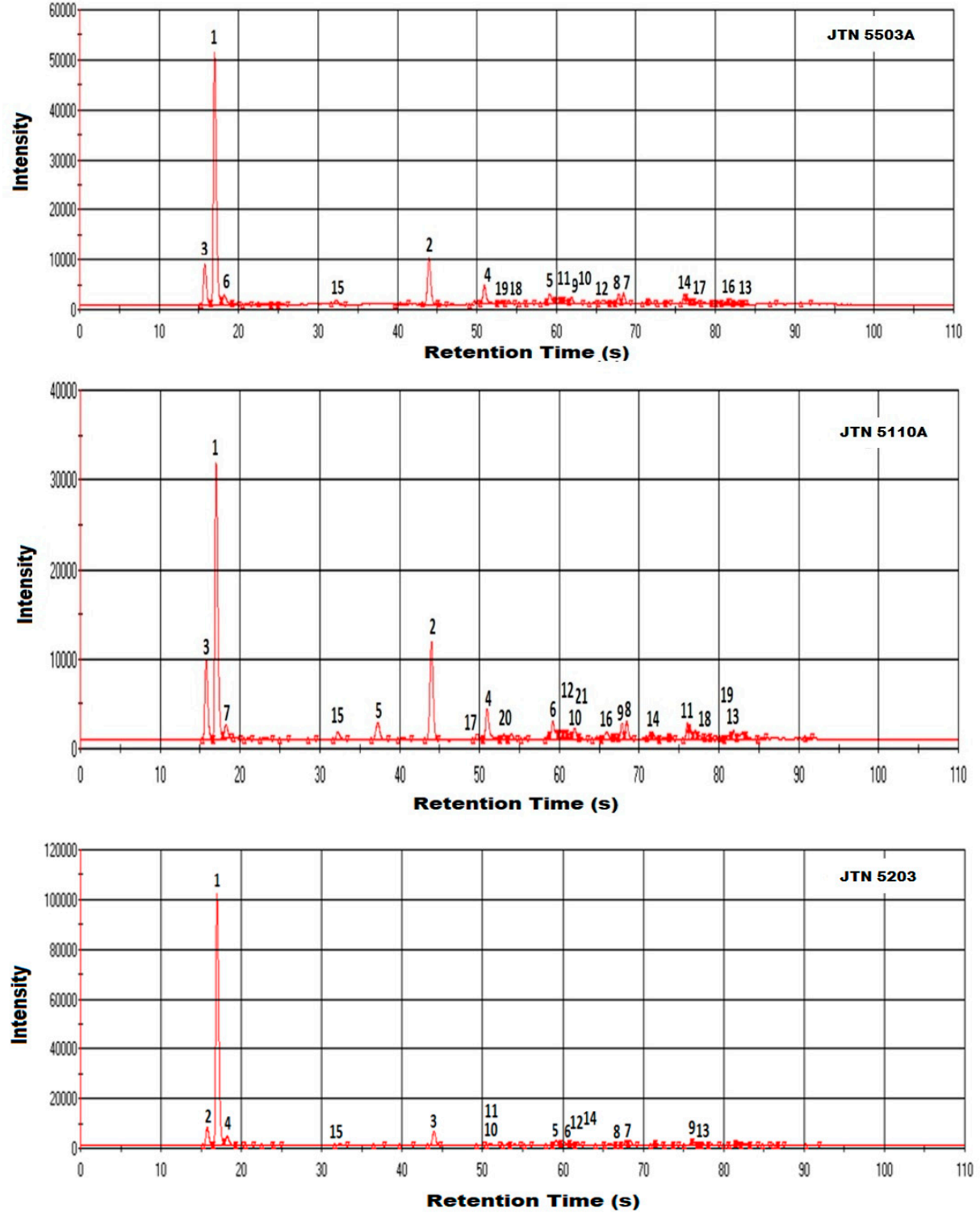

Figure 4. Ultra-fast GC Chromatogram of soybean varieties: JTN5503, JTN5110, JTN5203.

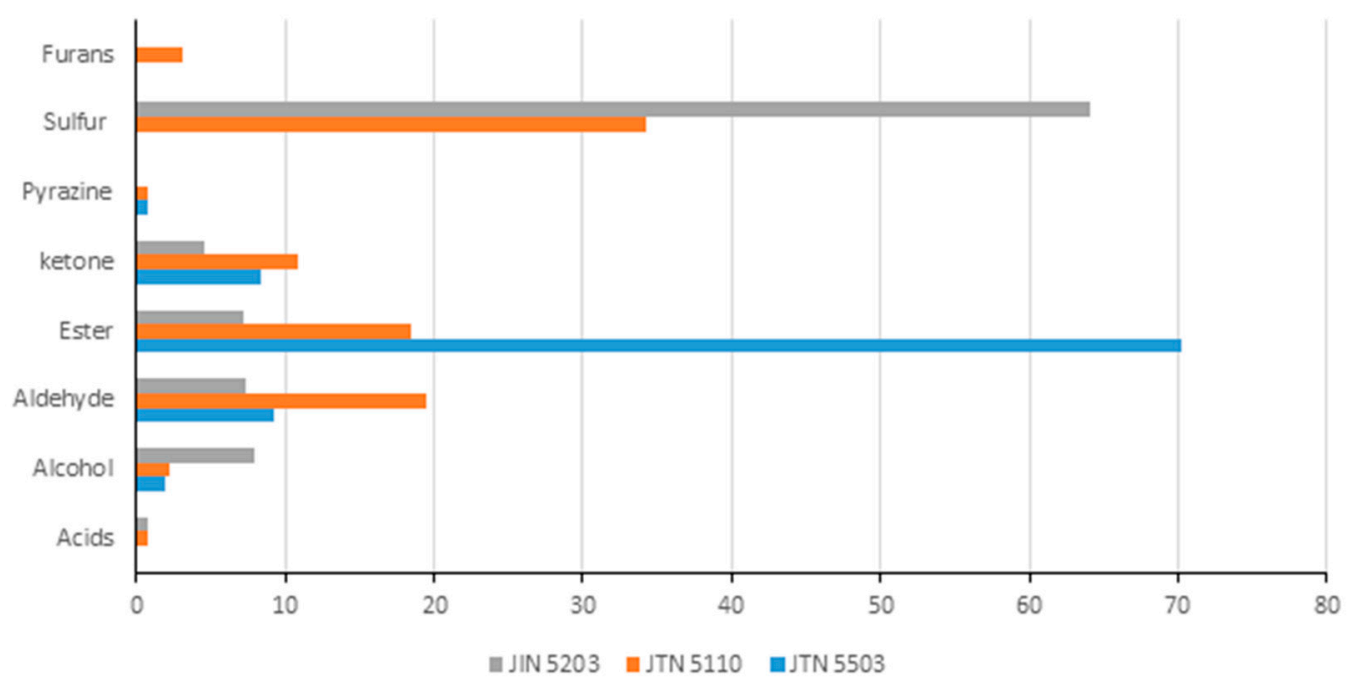

Figure 5. Comparison of volatile compounds in soybean varieties: JTN5503, JTN5110, JTN5203. 


\section{Conclusions}

E-nose has been used in a wide range of applications including odor analysis, quality control in food products, and biomedical applications. This study illustrates the use of e-nose as a versatile analysis tool and alternative method for measuring volatile compounds in soybean seeds with minimal sample preparation time. This approach can be used in a high-throughput phenotyping system and for screening different soybean lines. This system can be used as a rapid screening tool in breeding programs, in the selection of soybean mutants/varieties with different volatile profiles, and also for mapping the QTLs and loci responsible for these traits. This platform can also be used to link the beany flavor to seed volatile compounds, ultimately developing varieties with reduced off-flavor taste and better acceptance by the consumer.

Author Contributions: Conceptualization, A.T. and R.R.; methodology, R.R., D.K. and R.M.; validation, R.R. and A.T..; formal analysis, R.M. and D.K.; investigation, D.K. and R.R.; resources, A.T. and R.R. ; writing-original draft preparation, R.R., A.T. and D.K.; writing-review and editing, R.R. and A.T.; visualization, R.R. and D.K.; supervision, A.T. and R.R.; project administration, A.T.; funding acquisition, A.T.

Funding: This research was funded by the USDA National Institute of Food and Agriculture (Evans-Allen project), grant number 1005722 and Tennessee Soybean Board, grant number 16-123-P.

Acknowledgments: The USDA National Institute of Food and Agriculture, Evans-Allen project 1005722, Tennessee Soybean Board, project 16-123-P and Tennessee State University's College of Agriculture supported this work.

Conflicts of Interest: The authors declare no conflict of interest.

\section{References}

1. Lv, Y.-C.; Song, H.-L.; Li, X.; Wu, L.; Guo, S.-T. Influence of Blanching and Grinding Process with Hot Water on Beany and Non-Beany Flavor in Soymilk. J. Food Sci. 2011, 76, S20-S25. [CrossRef] [PubMed]

2. Lenis, J.M.; Gillman, J.D.; Lee, J.D.; Shannon, J.G.; Bilyeu, K.D. Soybean seed lipoxygenase genes: Molecular characterization and development of molecular marker assays. Theor. Appl. Genet. 2010, 120, 1139-1149. [CrossRef] [PubMed]

3. Yu, H.; Liu, R.; Hu, Y.; Xu, B. Flavor profiles of soymilk processed with four different processing technologies and 26 soybean cultivars grown in China. Int. J. Food Prop. 2017, 20, S2887-S2898. [CrossRef]

4. Kato, H.; Doi, Y.; Tsugita, T.; Kosai, K.; Kamiya, T.; Kurata, T. Changes in volatile flavour components of soybeans during roasting. Food Chem. 1981, 7, 87-94. [CrossRef]

5. Arai, S.; Noguchi, M.; Kaji, M.; Kato, H.; Fujimaki, M. n-Hexanal and Some Volatile Alcohols. Agric. Biol. Chem. 1970, 34, 1420-1423.

6. Fujimaki, M.; Arai, S.; Kirigaya, N.; Sakurai, Y. Studies on Flavor Components in Soybean. Agric. Biol. Chem. 1965, 29, 855-863.

7. Ram, M.S.; Seitz, L.M.; Rengarajan, R. Use of an Autosampler for Dynamic Headspace Extraction of Volatile Compounds from Grains and Effect of Added Water on the Extraction. J. Agric. Food Chem. 1999, 47, 4202-4208. [CrossRef] [PubMed]

8. Del Rosario, R.; de Lumen, B.O.; Habu, T.; Flath, R.A.; Richard Mon, T.; Teranishi, R. Comparison of Headspace Volatiles from Winged Beans and Soybeans. J. Agric. Food Chem. 1984, 32, 1011-1015. [CrossRef]

9. Seitz, L.M.; Sauer, D.B. Cereal Foods World; AACC International: St. Paul, MN, USA, 1992.

10. Boué, S.M.; Shih, B.Y.; Carter-Wientjes, C.H.; Cleveland, T.E. Identification of Volatile Compounds in Soybean at Various Developmental Stages Using Solid Phase Microextraction. J. Agric. Food Chem. 2003, 51, 4873-4876. [CrossRef] [PubMed]

11. Kaneko, S.; Kumazawa, K.; Nishimura, O. Studies on the Key Aroma Compounds in Soy Milk Made from Three Different Soybean Cultivars. J. Agric. Food Chem. 2011, 59, 12204-12209. [CrossRef] [PubMed]

12. Wilson, A.D.; Baietto, M. Advances in electronic-nose technologies developed for biomedical applications. Sensors (Basel) 2011, 11, 1105-1176. [CrossRef] [PubMed]

13. Arelli, P.R.; Young, L.D.; Mengistu, A. Registration of High Yielding and Multiple Disease-Resistant Soybean Germplasm JTN-5503. Crop Sci. 2006, 46, 2723. [CrossRef] 
14. Arelli, P.R.; Young, L.D.; Mengistu, A.; Gillen, A.M.; Fritz, L. Abstract: Newly Developed Conventional Soybean JTN-5110 Has Resistance to Multiple Pathogens. In Proceedings of the ASA, CSSA and SSSA International Annual Meetings, Long Beach, CA, USA, 2-5 November 2014.

15. Arelli, P.R.; Pantalone, V.R.; Allen, F.L.; Mengistu, A.; Fritz, L.A. Registration of JTN-5203 Soybean Germplasm with Resistance to Multiple Cyst Nematode Populations. J. Plant Regist. 2014, 9, 108. [CrossRef]

16. Chen, P.; Orazaly, M.; Wu, C.; Manjarrez-Sandoval, P.; Florez-Palacios, L.; Rupe, J.C.; Dombek, D.G.; Kirkpatrick, T.; Robbins, R.T. Registration of ‘UA 5014C' Soybean. J. Plant Regist. 2016, 10, 119. [CrossRef]

17. Chen, P. Soybean Cultivar UA 5414RR. U.S. Patent No. 9,326,478, 3 May 2016.

(C) 2019 by the authors. Licensee MDPI, Basel, Switzerland. This article is an open access article distributed under the terms and conditions of the Creative Commons Attribution (CC BY) license (http://creativecommons.org/licenses/by/4.0/). 